\title{
Range-based intensity normalization of ALS data over forested areas using a sensor tracking method from multiple returns
}

\author{
Jean-Romain Roussel ${ }^{\mathrm{a}, 1, *}$, Jean-François Bourdon ${ }^{\mathrm{b}}$, Alexis Achim ${ }^{\mathrm{a}}$ \\ ${ }^{a}$ Centre de recherche sur les matériaux renouvelables, Département des sciences du bois et de la forêt, Université Laval, Québec, QC, Canada \\ ${ }^{b}$ Direction des inventaires forestiers, Ministère des Forêts, de la Faune et des Parcs du Québec, QC, Canada
}

\begin{abstract}
Airborne laser scanning (ALS) point-clouds are used in forest inventory to map properties of the resource. In most cases, only the $(x, y, z)$ coordinates of the point cloud are used to build predictive models of forest structure. Despite being recorded and provided by data suppliers, the intensity values associated with each point are rarely used as an input to such models because raw intensity values vary within and between datasets not only due to variations of the target reflectivity, but also due to variations in settings and conditions that may either modify the emitted energy or the distance between the sensor and the targets. Some studies have proposed data-driven methods of normalization, but these are often impossible to apply in practice because of the need to acquire additional reference data. Other studies have proposed model-driven methods of normalization, but in this case the difficulty of application lies with need to know the position of the sensor at any given time during the survey. In this study we applied a method to track the sensor position using pulses associated with multiple returns, which was then used to apply a model-driven correction of the intensity values within several datasets. This normalization method is based only a simple model-driven equation and does not require any reference data, thus making it applicable to any dataset. Our results demonstrate the very high accuracy of the sensor positioning method with an error under $0.5 \%$. Using this information, the model-driven range correction then performed a satisfactory normalization of intensities within different datasets. We provide an open-source and ready-to-use tool to facilitate the application of the normalization method, which in turn could promote a better use of intensity values in ALS studies.
\end{abstract}

Keywords: ALS, Intensity, Range Correction, Sensor Tracking

\section{Introduction}

Airborne light detection and ranging (or ALS for airborne laser scanning) technology is widely used for land and vegetation surveys in forestry and other environmental applications. It provides end-users with a point cloud that is a three-dimensional representation of the structure of the landscape. To be of practical use, such point clouds must be processed using algorithms that convert the raw data into information that can be interpreted, which is then typically output as a map. In forestry applications, the most commonly used information is the three-dimensional coordinates $(x, y, z)$ of the points, which can be interpreted with relative ease to derive information describing the structure of the forest such as individual tree heights, height of the forest canopy, light penetration into the canopy, etc. However, ALS point clouds also contain several more dimensions of information including the recorded intensity of each point, an information that is usually supplied by data providers (Höfle and Pfeifer, 2007).

\footnotetext{
* Corresponding author

Email address: jean-roma in.roussel. 1@ulaval. ca (Jean-Romain Roussel)
}

The value of intensity of each point is associated, at least partially, with surface reflectivity of the target, so it can be used to discriminate objects or structures in the landscape. Current knowledge on the physical aspects of the variations in intensity has been synthesized by e.g. Hopkinson (2007); Kaasalainen et al. (2007); Vain et al. (2009, 2011); Wagner (2010); Gatziolis (2013), so here we chose to focus on the practical applications.

Previous studies have shown that intensity values can contribute to species identification (Kim et al., 2009; Korpela et al., 2009; Zhang and Liu, 2013), land-cover classification (Liu, 2008), forest attributes modelling (Bright et al., 2013; Sumnall et al., 2016), snag detection (Yao et al., 2012; Wing et al., 2015) or ground point filtering (Yunfei et al., 2008). However, despite such promising results, intensity is rarely used in practice because measured values do not only vary depending on the reflectivity and size of the targets, but also on the flying altitude, atmospheric conditions and laser settings (Baltsavias, 1999; Wagner, 2010). Therefore, intensity values may be very different between datasets due to variations in the devices used, weather conditions during the acquisition, emitted pulse rates, storage modes or any other settings that may either modify the emitted energy 
or the distance between the sensor and the targets. This reality limits the relevance of raw intensity values for any applications requiring transferable processing workflows.

Within a single survey, this information could be expected to be reliable if only one sensor and one set of settings was used. However, the raw intensity data is still rarely usable because the distance between the sensor and the targets (or range) is not constant all along the survey. Range $(R)$ is considered the most important source of energy loss during the travel of the beam through the atmosphere (Hopkinson, 2007; Kaasalainen et al., 2007; Gatziolis, 2013). This fact, which has been widely discussed in previous studies, stems from the physical processes known to drive energy transfer, and which have led to the RaDAR equation (Hopkinson, 2007; Kaasalainen et al., 2007; Vain et al., 2009; Wagner, 2010). In any given survey, $R$ variations occur because the aircraft does not (or cannot) fly perfectly straight at a constant altitude, and even if it could, the topography of the landscape introduces variations of distance between the sensor and the targets. Consequently, intensity values are in reality a mixed record of target reflectivity, target size, topography and sensor altitude (fig. 1). A lack of ability to dissociate these sources of variation has hindered the use of intensity values in ALS studies.

To be used more adequately, intensity must be corrected and normalized. Modern ALS systems now provide real-time range normalization of intensity values; however, this is based on undocumented proprietary routines, which complicates comparisons between datasets and limits our capacity to conduct reproducible studies. More importantly in the context of the current study, there remains a wealth of older datasets still being used and in which intensity values were not corrected at the source. The simplest option to normalize these values is to use the so-called model-driven 'range correction', which accounts for the fact that beams do not travel the same distance from sensor to target (equation 1).

$$
\hat{I}=I\left(\frac{R}{R_{s}}\right)^{f}
$$

where $\hat{I}$ is the normalized intensity equivalent to the intensity that would have been recorded if all points had been recorded with a constant range, $I$ is the observed intensity, $R$ is the distance between the laser instrument and the returns, $R_{s}$ is an arbitrary reference distance and the exponent $f$ represents the rate of energy attenuation sustained by the pulse as it travels through a medium back and forth from a target. The value of $f$ depends on the surface type and the theory states that a value of 2 corresponds to homogeneous targets filling the full pulse footprint, a value of 3 corresponds to linear objects (e.g. power line wires), and a value of 4 corresponds to individual large scatterers (Kaasalainen et al., 2007). This value is therefore likely to vary between 2 and 3 in a vegetated context (Korpela, 2008; Gatziolis, 2013).
Other model-driven range corrections have been proposed by Höfle and Pfeifer (2007); Vain et al. (2009), which include equation 1 but also take into account other variables such as the emitted energy and atmospheric transmittance. However, Gatziolis (2013) showed that the simple range correction described in equation 1 performs accurately, a fact also supported by its successful application in other studies (e.g. Yoga et al., 2017; Donoghue et al., 2007; García et al., 2010). Despite this, a strong limitation of the approach comes from the requirement to know the range $R$ at any instant $t$. Such data may in some cases be supplied by the data provider, but currently this is not common practice. Neither Gatziolis (2013), nor Yoga et al. (2017), nor Höfle and Pfeifer (2007) explicitly stated how they accessed this information in their respective studies, but we may assume that they had access to the position of the aircraft recorded by the embarked positioning system. Donoghue et al. (2007) and García et al. (2010) bypassed the problem by "approximating the range of each point as the difference between the average altitude of the flight and the altitude of each point". While this is an acceptable solution for comparisons between surveys (all else being equal), it does not account for the within-survey variation of sensor altitude, which can be important, as shown in (fig. 1).

In the absence of reference data, some authors have opted for a data-driven correction. Höfle and Pfeifer (2007) developed a correction approach that empirically estimates the parameters of an overall correction function using a least-squares adjustment. To be relevant, however, this approach requires homogeneous areas flown at different altitudes to derive the parameters of the function. Beyond the specificities of variants of this approach, data-driven corrections have to rely on both reference and calibration data, which similarly to the sensor tracking, are often non available.

In the absence of practically applicable correction methods, several studies have used raw intensity values as a predictor variable in statistical modelling efforts (e.g. Yunfei et al., 2008; Zhang and Liu, 2013; Wing et al., 2015; Sumnall et al., 2016). Statistically significant relationships have been reported, but we can only assume that they came from ideal cases where little variation occurred within and between flightlines. In several other studies, raw intensity was found to be a non significant variable in prediction models (e.g. Moffiet et al., 2005; Popescu and Zhao, 2008). It cannot be assumed that a range correction would have made intensity a significant variable, but it provides at least one plausible explanation for the absence of relationship, as mentioned in Moffiet et al. (2005). In addition, the publication bias towards significant relationships (e.g. Dickersin, 1990) may explain the limited number of instances in the literature where authors have reported attempts to use intensity-based metrics. 

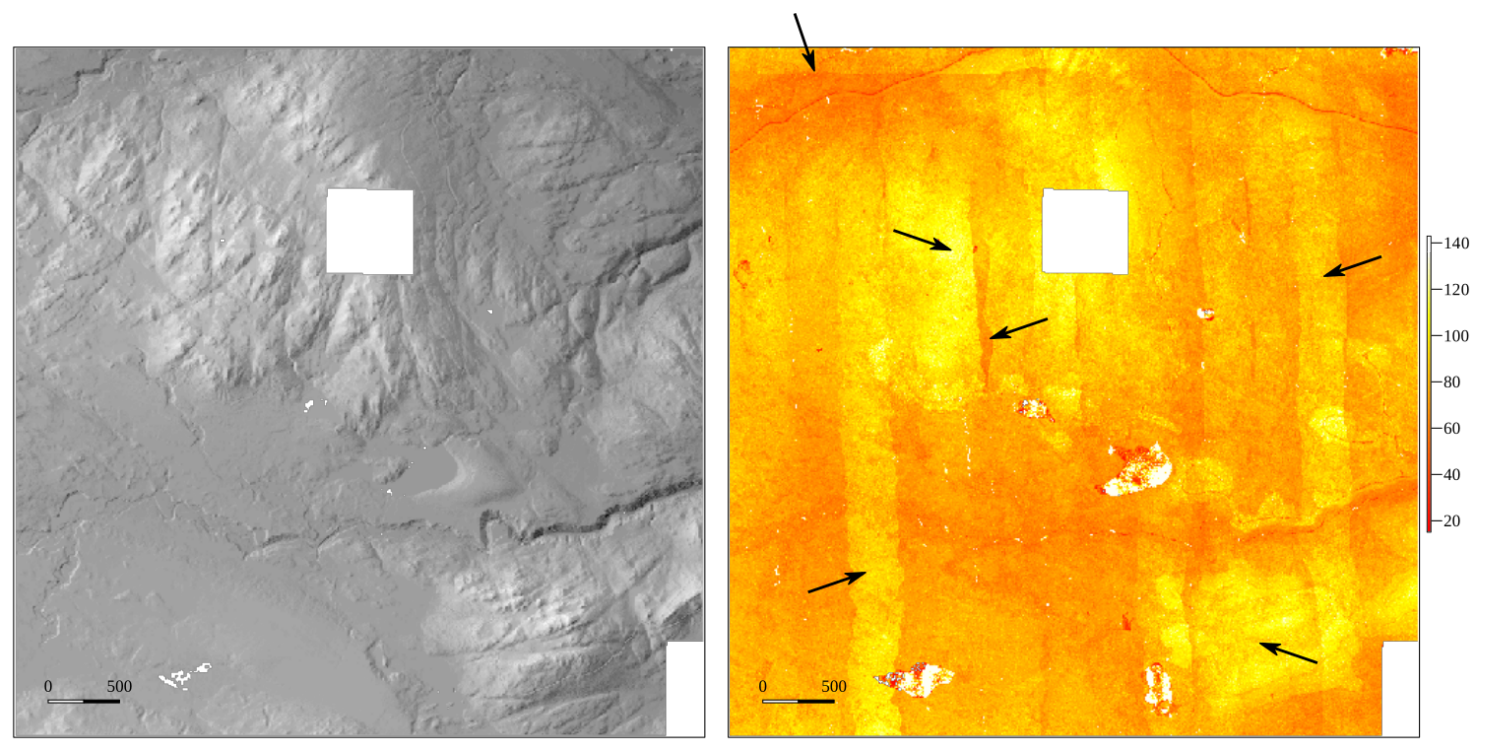

Figure 1: Left: hill-shaded image of the topography. Right: images of the maximum raw intensity of first returns within $5 \times 5$-m cells. Arrows show some obvious variations of intensity that do not correspond to any land structures but instead are pure artifacts of the sensor's altitude and the topography. They indicate some flightlines and some regions of high elevation (note the transversal flightline that can be observed at the top of the image). In this map, the reflectivity properties of the targets are mostly hidden by other effects, and thus the intensity values are non-usable. The only clearly visible structure is a road. Blank regions correspond to the absence of data, either removed by the provider or from water bodies.

In this paper, we propose a method to produce a range-based intensity normalization in ALS data by reconstructing the sensor trajectory. Using the alignment of multiple returns, we were able to apply an accurate range correction in different datasets based on the method proposed by Gatziolis (2013), a process that also allowed us to retrieve optimal $f$ values. Our workflow is based on algebra and model-driven equations, and does not require any reference or validation data. We have provided all the algorithms and processing methods as an open-source, straightforward and ready-to-use tool in an ALS-dedicated software, with the intention to enable researchers and other users to include intensity-based metrics in their analyses and to reproduce this work easily. We demonstrate the validity and the accuracy of the sensor-tracking method, and its applicability to perform a range correction within a dataset. In doing so, we also confirmed the reproducibility and validity of a previous study related to intensity normalization with a range correction (Gatziolis, 2013).

\section{Material and methods}

\subsection{ALS data}

ALS datasets from three locations were used for this study: Québec, Canada; British Columbia, Canada and California, USA (datasets numbered \#1 to \#5 table 1). Two datasets from Québec (\#1 and \#2) were associated with the gps track of the aircraft given by the data provider, while the other datasets represented the typical situation where such information is not provided (\#3 to \#5). The datasets associated with the aircraft tracks were used to validate and estimate the accuracy of the sensor tracking method. The other regular datasets were used to apply a range correction of intensities using our sensor tracking method. The dataset from California comes from publicly available data provided by the U.S. Geological Survey (U.S. Geological Survey, 2011).

From each regular dataset that did not contain gps tracking data, we extracted two arbitrary regions of interest of approximately 5 to $10 \mathrm{~km}^{2}$. These regions of interest were coded $\# i .1$ and $\# i .2$. The choice of regions and their sizes were purely driven by pragmatic needs. We searched for regions of interest showing large variations of intensity to test the method in the worst possible conditions, and chose small enough areas to ensure that land structures remained well visible when printing the figures for this article. The same needs led us to use the Californian dataset. Among the large number of publicly available datasets, we randomly selected one that was in a mountainous region.

A summary of the datasets is given in table 1. Overlap is described as "non available" for the dataset used to test the sensor tracking accuracy because the flight pattern consists of a set of two orthogonal acquisitions. Flightlines overlapped each other more than once, and in any case these datasets were only used to track the position of the sensor, so the processing was equivalent to treating each flightline individually. This also implied that the point density given for these datasets is given by flightline. The "tracking density" shows the pulse density actually used to track the sensor. The 
sensor tracking method described in section 2.2 uses only multiple returns, and consequently relies on roughly $30 \%$ of the data $(70 \%$ of the points typically consisting of single returns). We also decimated the dataset by selecting only one pulse every 0.0001 second to accelerate the computation.

The range correction was not performed on \#1 and \#2 because the intensity had already been normalized by the data provider. Intensity maps for these datasets were already very clean without any visible effects of the flightlines or of the topography. The Riegl VQ-780i sensor can indeed perform real time range correction. Finally, the sensor used in the Californian survey was not documented in the metadata provided along with the point cloud, so it is presented as non available in table 1.

\subsection{Sensor trajectory}

When several returns from a single pulse are detected, the sensor computes their positions as being in the centre of the footprint, which implies that they are all aligned. A line drawn between and beyond a series of returns should therefore intersect the sensor position. Several consecutive pulses emitted in a very short time interval can thus be used to approximate an intersection point in the sky that corresponds to the sensor position at the time of sampling, given that the sensor carrier has not moved much if the interval is short enough.

Using first and last returns, we computed the euclidean vector of each pulse that returned multiple returns. We grouped these pulses in bins of 0.5 seconds and computed the position of the intersection of the lines in the sky to get a sensor position at a time resolution of 0.5 seconds (figure 2). At $240 \mathrm{~km} / \mathrm{h}, 0.5$ seconds corresponds to $30 \mathrm{~m}$ bins. The time interval may be increased or decreased as a function of the moving speed and as a function of the required target accuracy. Here, we used a time resolution twice as high as the tracks given by the data provider of dataset \#1 and $\# 2$, which were given with a time resolution of 1 second for the same nominal aircraft speed. In reality, the lines did not precisely intersect each other both because the sensor was moving and because of the inaccuracies introduced at different levels of the digitization. Consequently, there could be no analytical solution to determine the intersection point. Instead, we found the point that was mutually closest to all lines in a least-squares sense. In each bin, we computed the intersection with a weighted least squares to approximate the intersection point of all lines (figure 2). The least squares were weighted by the distance between the first and last return, so that multiple returns that were near each other accounted for less than those that were more distant.

\subsection{Sensor trajectory accuracy assessment}

In dataset \#1 and \#2, after computing the sensor track from multiple returns we compared with the references given by the data provider. The references were given at

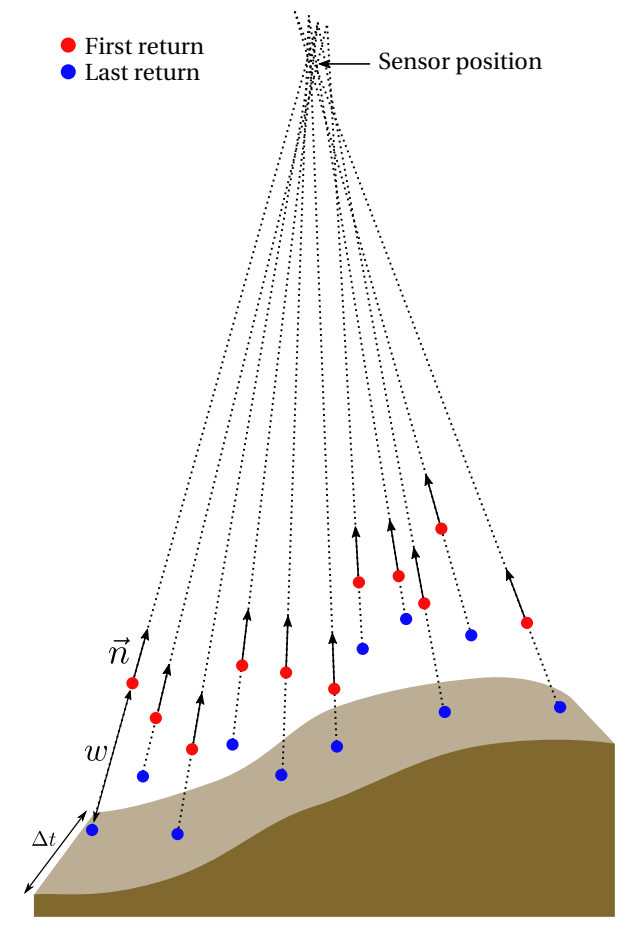

Figure 2: Schematic representation of a bin of ALS data acquired within a short time-frame $\Delta t$. First and last returns are paired by pulse and euclidian vectors $\vec{n}$ are computed. The least square method was applied to approximate the sensor position with a weight $w$ applied to each vector as a function of the distance $d$ between the first and last returns.

every second while our computed positions were at every 0.5 seconds, and not necessarily in a synchronized multiple of the reference times. Consequently we applied a linear interpolation of the trajectory to compute the positioning of the sensor at the exact same times as the reference. We then computed the errors $\Delta x, \Delta y$ and $\Delta z$ of the positions in each direction and estimated the root mean square differences (RMSD) of the errors in each direction. We compared approximately 5000 and 30000 sensor positions over $300 \mathrm{~km}^{2}$ in datasets \#1 and \#2, respectively.

\subsection{Intensity normalization}

After computing the sensor track from multiple returns in datasets \#3, \#4 and \#5, the intensity of each point was corrected using the range correction provided by equation 1. The impulsion time of each point was used to retrieve the position of the sensor at this time using the sensor trajectory computed according to the method described in section 2.2. This method provided the positioning at discrete instants $t_{i}$, but a linear interpolation was performed to provide a position at any intermediate time $t$.

The reference range $R_{s}$ being an arbitrary value, the range-normalized intensity $\hat{I}$ also provides an arbitrary value. In an attempt to produce values of $\hat{I}$ with a similar range to the original values given by the data provider, $R_{S}$ 


\begin{tabular}{rcc|ccc}
\multicolumn{4}{c}{ Sensor tracking } & \multicolumn{3}{c}{ Range correction } \\
\hline \hline Code & $\# 1$ & $\# 2$ & $\# 3$ & $\# 4$ & $\# 5$ \\
\hline Location & Québec & Québec & Québec & B. Columbia & California \\
Elevation $(\mathrm{m})$ & $740-950$ & $740-950$ & & & \\
$\# i .1$ & & & $530-940$ & $150-440$ & $140-400$ \\
$\# i .2$ & & & $570-790$ & $250-470$ & $1200-1600$ \\
Tracking density (m $\left.{ }^{-2}\right)$ & 0.6 & 0.6 & 0.4 & 0.7 & 0.4 \\
\hline Sensor & VQ-780i & VQ-780i & ALTM 3100 & ALTM 3100EA & NA \\
Altitude AGL (m) & 2000 & 1000 & 1000 & 700 & 1000 \\
Speed (km/h) & 240 & 240 & 240 & 240 & 240 \\
Overlap (\%) & NA & NA & 50 & 50 & 20 \\
Point density (m $\left.{ }^{-2}\right)$ & $1-2$ & $11-13$ & $6-7$ & $11-12$ & $8-9$ \\
\hline
\end{tabular}

Table 1: Summary of the location, topography as well as aircraft and sensor configuration for the datasets used in this study. For \#1 and \#2 overlap and density are given by flightline. The tracking density is the pulse density that was used to compute the sensor positions.

must be chosen to be close to the average original range. Following Gatziolis (2013), we thus defined $R_{s}$ as equal to the average of the computed sensor altitudes minus the average elevation of the terrain.

After defining $R_{S}$ and obtaining the $R$, we still required to estimate $f$ to compute equation 1. A value of 2 is often used by default but Gatziolis (2013) found optimal values in the range of $2.1-2.5$ over forests. We consequently searched for optimal $f$ values.

\subsection{Optimal $f$ values}

Following Gatziolis (2013) we used the redundant informations in overlaps to estimate a value of $f$ in each region of interest. The original idea of Gatziolis (2013) was to pair very close pulses from different flightlines. The paired pulses were assumed to hit the same object so that the major part of their intensity difference would be indicative of a difference of range. Gatziolis (2013) estimated the value of $f$ as the one that minimized the RMSD of paired, range-normalized intensities (equation 2):

$$
\sqrt{\frac{1}{n} \sum_{i=1}^{n}\left(I_{i, 1}\left(\frac{R_{i, 1}}{\bar{R}}\right)^{f}-I_{i, 2}\left(\frac{R_{i, 2}}{\bar{R}}\right)^{f}\right)^{2}}
$$

where $n$ is the number of return pairs identified, $I_{i, 1}$ and $I_{i, 2}$ are the observed intensities of each return in a pair, $R_{i 1}$ and $R_{i, 2}$ are the corresponding ranges, and $\bar{R}$ is the mean range among all paired returns.

This method is relatively complex since it requires to pair pulses and compute the difference for each pair. We opted for a simplification by pairing $5 \times 5$ meter pixels instead of pulses. Our method still relied on equation 2 but the meaning of the terms were adjusted to: $n$ being the number of pairs of pixels, $I_{i, 1}$ and $I_{i, 2}$ the observed derived metrics of intensities (mean, max) in the pixels, $R_{i, 1}$ and $R_{i, 2}$ the corresponding average ranges within the pixels and $\bar{R}$ the mean range among all returns. The two methods are actually closely equivalent. While Gatziolis (2013) assumed that two paired pulses should return the same intensity, we assumed that two pixels should return the same derived metrics of intensity. This also implied an assumption that the range within a $5 \times 5 \mathrm{~m}$ pixel is constant, which appeared reasonable.

We computed equation 2 for $f \in[2.0,2.1,2.2, \ldots, 3.0]$. The best $f$ was the one that minimized the RMSD of the differences i.e. the one that provided, on average, the same metrics in each flightlines. $f_{1}$ was defined as the $f$ value that minimized the RMSD of the average intensity while $f_{2}$ minimized the RMSD of the maximum intensity. The best $f$ was computed as the average of $f_{1}$ and $f_{2}$.

\section{Results}

\subsection{Sensor trajectory accuracy assessment}

In the sensor trajectory computation, the RMSD of the positioning on $x$ and $y$ was under 5 meters in both datasets $\# 1$ and \#2. Even at a low altitude, the effect of such an error on $(x, y)$ would only bring an error of a few centimetres on the estimated range $R$ i.e. less than $0.01 \%$. The RMSD of the positioning on the $z$ elevations was under $10 \mathrm{~m}$ in both datasets. This is meaningful only relatively to the elevation of the sensor since it is the error that most directly affects the estimated range. With an elevation of $2000 \mathrm{~m}$ above ground level, such an error would correspond to an order of magnitude of less than $0.5 \%$. Figure 3 shows the distribution of the absolute errors on $x y$ (euclidean distance) in meters and the distribution of the errors on $z$ in \%. Thirteen comparisons out of 4600 in dataset \#1 and 89 out of 30120 in dataset \#2 were above $\pm 2 \%$ of error. Figure 3 had to be cropped at $\pm 1 \%$ for readability.

\subsection{Optimal value of $f$}

Different optimal values of $f$ were found in each region of interest and variations occurred not only between but also within our three ALS datasets. Table 2 summarizes 

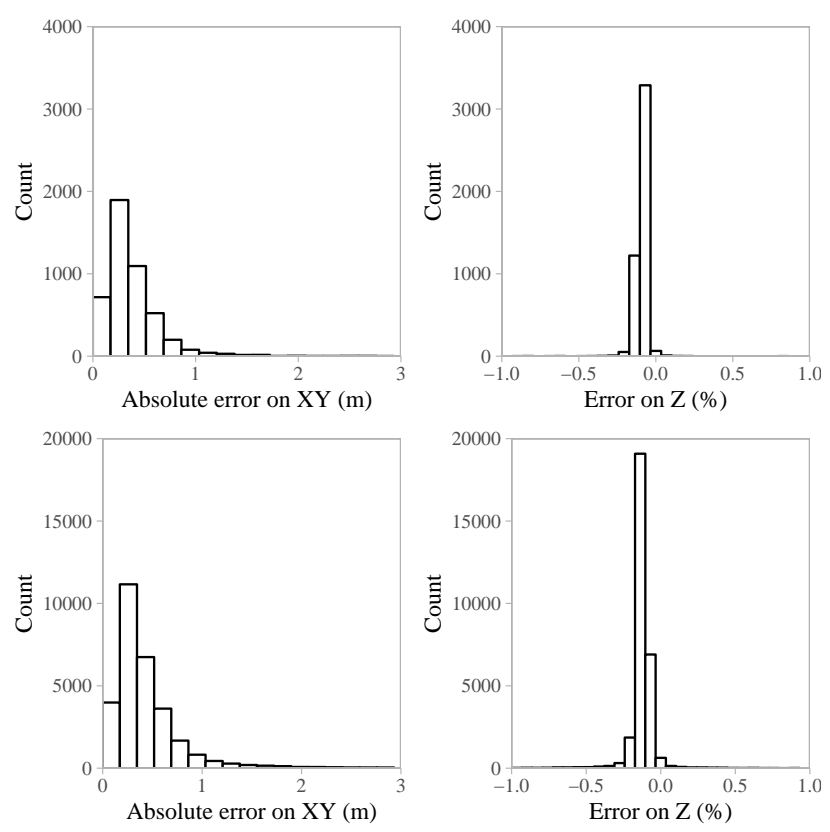

Figure 3: Distribution of absolute errors of sensor positioning on the $x y$ plane (euclidian distance to the sensor) and error on the $z$ axis for datasets \#1 and \#2 (table 1).

the obtained values and the number of pixel pairs used to estimate them.

\begin{tabular}{ccc}
\hline Data & $n$ & $f$ \\
\hline \hline$\# 3.1$ & 182000 & 2.50 \\
$\# 3.2$ & 142000 & 2.15 \\
$\# 4.1$ & 191000 & 2.10 \\
$\# 4.2$ & 169000 & 2.15 \\
$\# 5.1$ & 384000 & 2.55 \\
$\# 5.2$ & 537000 & 2.60 \\
\hline
\end{tabular}

Table 2: Optimal values of $f$ found in each region of interest (see table 1). $n$ is the number of pixel pairs used to estimate these values

\subsection{Intensity normalization}

Figures 4, 5 and 6 show, for each regions of interest, the images of $I_{\max }$ and $\hat{I}_{\max }$ with a resolution of $5 \mathrm{~m}$. For space considerations, we only displayed the maximum intensity values because they are usually affected by a single flightline (the one where the sensor was the lowest), which thus makes flightline artifacts more clearly visible on the images. Conversely, the mean intensity is the average of the overlapping flightlines, which tends to homogenize the artifacts, especially when overlaps are $50 \%$. All figures are provided in the supplementary materials along with a digital terrain model, a shaded digital terrain model and the images of $I_{\text {mean }}$ and $\hat{I}_{\text {mean }}$ for a better appreciation of the results.

In the absence of a reference, it is not possible to evaluate how good the correction was. However, it can be observed that all visible artifacts were removed by the correction. The effects of hill and valley topography, easily visible on the original images, were removed. Flightlines are no longer visible on the corrected images and obvious land structures such as clearcuts belong in the same range of intensities.

\section{Discussion}

\subsection{Sensor tracking and normalization accuracy}

Results showed that our sensor tracking method can be considered as very accurate with an estimated error $\delta$ below $0.5 \%$ on $R$. The impact of this positioning error then has to be assessed in terms of corrected intensity range i.e. $\hat{I}(1 \pm \delta)^{f}$. For $I=100, R=1200, R_{s}=1000, f=2.3$ and $\delta=0.3 \%$, which was the typical $95^{\text {th }}$ percentile of error in our datasets, we obtain a normalized intensity $\hat{I} \in[151.05,153.1]$ rounded to $[151,153]$ because las files store intensity as 16-bit integers. This variation represents approximately $\pm 2 \%$ of the applied correction, on average.

The range correction method we used inherently takes into account the effect of scan angle, which is important because a scan angle of $20^{\circ}$ increases the range by $6 \%$. Some authors proposed a variation of equation 1 to explicitly include the effect of scan angle $\theta$ (e.g. Hopkinson, 2007) (equation 3).

$$
\hat{I}=I\left(\frac{R}{R_{s} \cos (\theta)}\right)^{f}
$$

This equation applies to situations where $R$ is the vertical range and the surveyed area is flat. However, in practice the topography of the terrain implies that the actual range at angle $\theta$ is not necessarily $\frac{1}{\cos (\theta)}$ longer than the range at $0^{\circ}$. When computing the range for each point using the distance to the aircraft position (i.e. using equation 1) the effects of topography, aircraft altitude and scan angle are all taken into account.

\subsection{Limits of the normalization}

An important limitation of our approach is that the applied range correction does not return absolute values representative of the physical properties of the targets. First, it applies a relative correction to raw measurement values for which the physical units are unknown. Second, in the absence of information on sensor specifications, such as the emitted energy, or on the digitization process operated by the data-provider at the hardware or software levels, it is not possible to retrieve the physical meaning of the (raw or corrected) intensity values. Third, the returned intensity is a function of both the target reflectance and the illuminated area. In the absence of information on either of these variables, we face a ill-defined problem for which it is not possible to disentangle the effects of the reflectance and/or the area. Intensity values in ALS data must therefore be taken for what they are: dimensionless values that are not physically meaningful per se, but that may still provide 

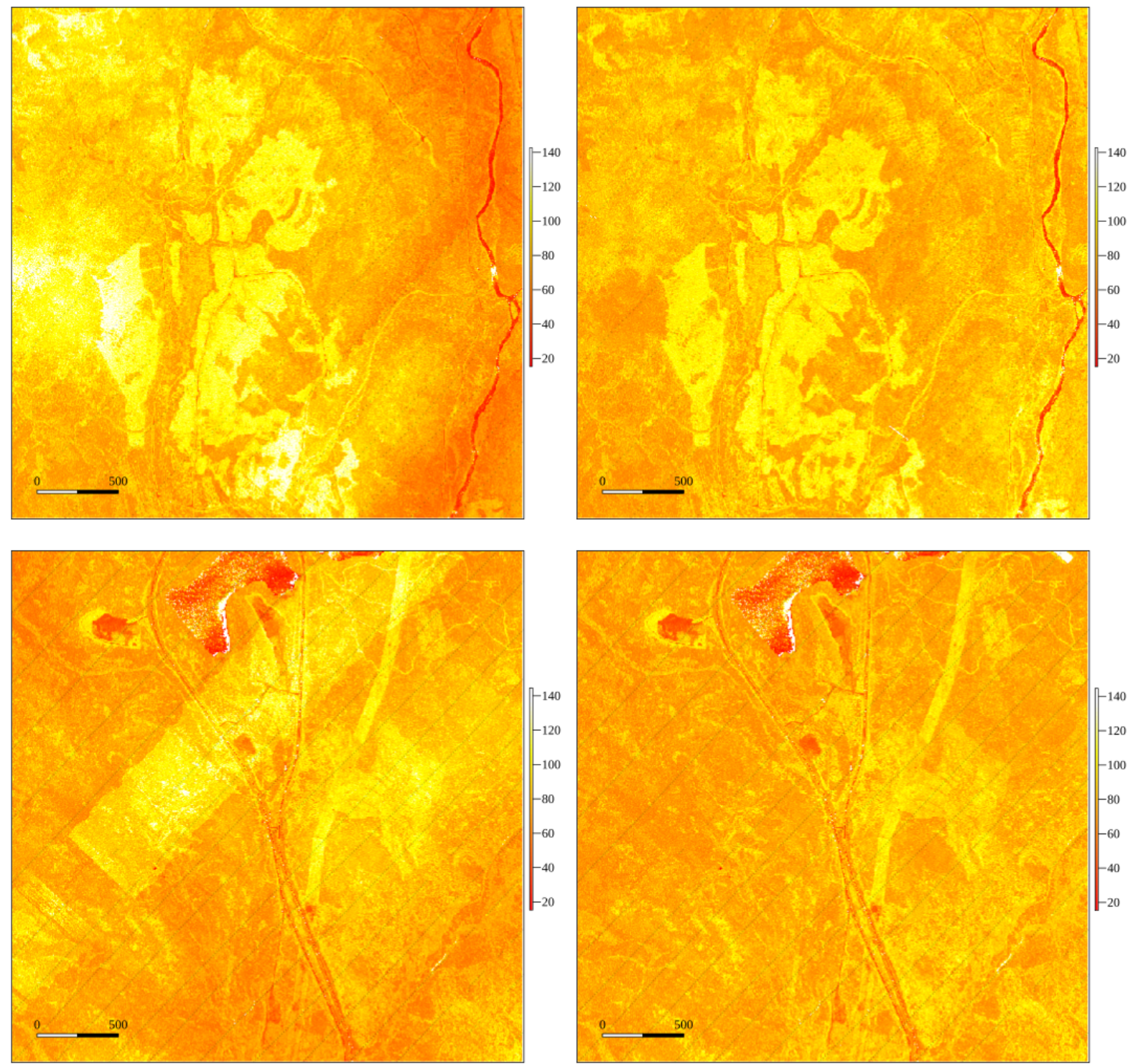

Figure 4: Images of the maximum intensity within $5 \times 5 \mathrm{~m}$ cells for raw intensities (left) and range-normalized intensities (right) for regions of interest \#3.1 and \#3.2 (table 1). Narrow and almost transparent lines correspond to the sensor trajectory reconstructed. On the left hand side, the effects of topography and flightlines are clearly visible and one can see some obvious incoherence. On the right hand side these artifacts are no longer visible. Intensity correction also revealed land structures that were previously hidden in the noise generated by flightlines and topography. The narrow bright strip in \#3.2 is not a flightline but a transmission line's rights-of-way.

interesting additional information that can be related to some characteristics of the surveyed area.

The range correction method proposed in this study therefore remains relative and specific to a given dataset. It also assumes that the survey was completed within a short time frame, with the same weather conditions and a single sensor. Its only purpose is to remove the variation in intensity values that are attributable to aircraft position and topography. Our example datasets showed that this variation can be important. The proposed correction method may thus prove useful to make use of intensity values for applications such as vegetation type assessments or the detection of features such as water bodies, roads, etc. (Kim et al., 2009; Korpela et al., 2009; Zhang and Liu, 2013; Liu, 2008; Bright et al., 2013; Sumnall et al., 2016; Yao et al., 2012; Wing et al., 2015; Yunfei et al., 2008).

Making multi-dataset analyses comparable, such as those generated by multi-temporal surveys, would require another strategy. In this case, intensity values would need to be converted to relative, comparable measurements for different datasets sampled with different ALS systems and in different atmospheric conditions (Höfle and Pfeifer, 2007). 

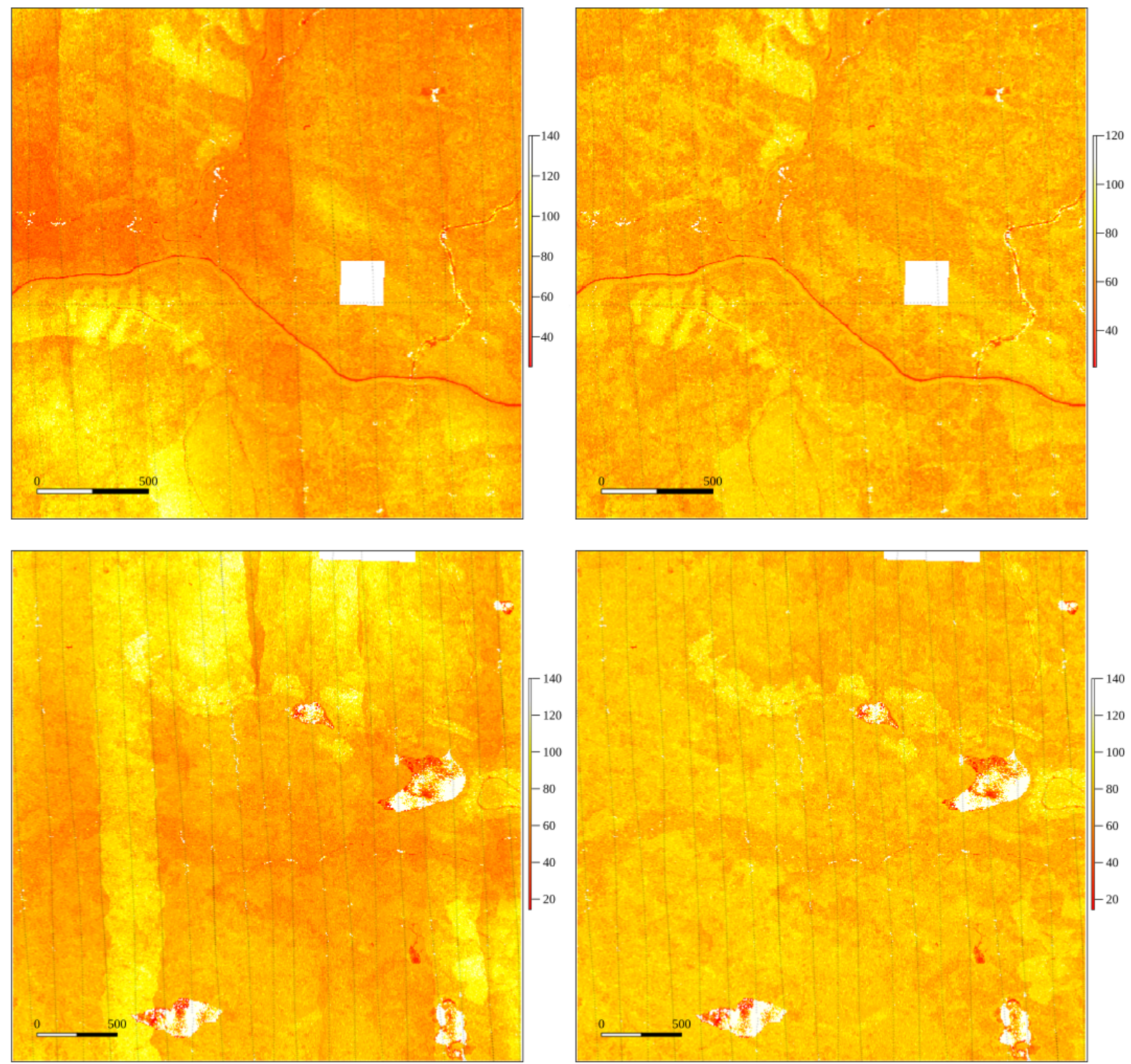

Figure 5: Images of the maximum intensity within $5 \times 5 \mathrm{~m}$ cells for raw intensities (left) and range-normalized intensities (right) for regions of interest \#4.1 and \#4.2 (table 1). Narrow and almost transparent lines correspond to the sensor trajectory reconstructed. On the left hand side, the effects of topography and flightlines are clearly visible and one can see some obvious incoherence. On the right hand side these artifacts are no longer visible. Intensity correction also revealed land structures that were previously hidden in the noise generated by flightlines and topography.

In a multi-dataset context, Yao et al. (2012) applied the range correction 'as-is' but raw intensity values were first corrected by emitted energy and all the coverages were sampled with the same sensor, which is not representative of the general case. Provided that good within-survey corrections are performed that exclude dummy effects of sampling design and effects of topography, multi-temporal analyses could be normalized by matching the intensity distribution (histogram matching) in invariable structures such as roads, for example.

The broad coverage areas that are surveyed by different data providers, and thus with different devices and settings, bear similar issues. In this case we suggest using overlapping regions for matching once within-survey corrections have been applied. The method we provide in this study could therefore contribute towards resolving issues of survey-to-survey intensity normalization.

\subsection{Optimal $f$ values over forested areas}

The optimal value of $f$ was found to be larger than 2 but smaller than 3 in each trial dataset. These values are similar to those reported by Gatziolis (2013) or Korpela (2008), but contrary to Gatziolis (2013), we did not find that the quality of the normalization was highly dependent on the value of $f$. Although we reported computed optimal values, we could not visually detect any differences in figures 4,5 or 6 when 

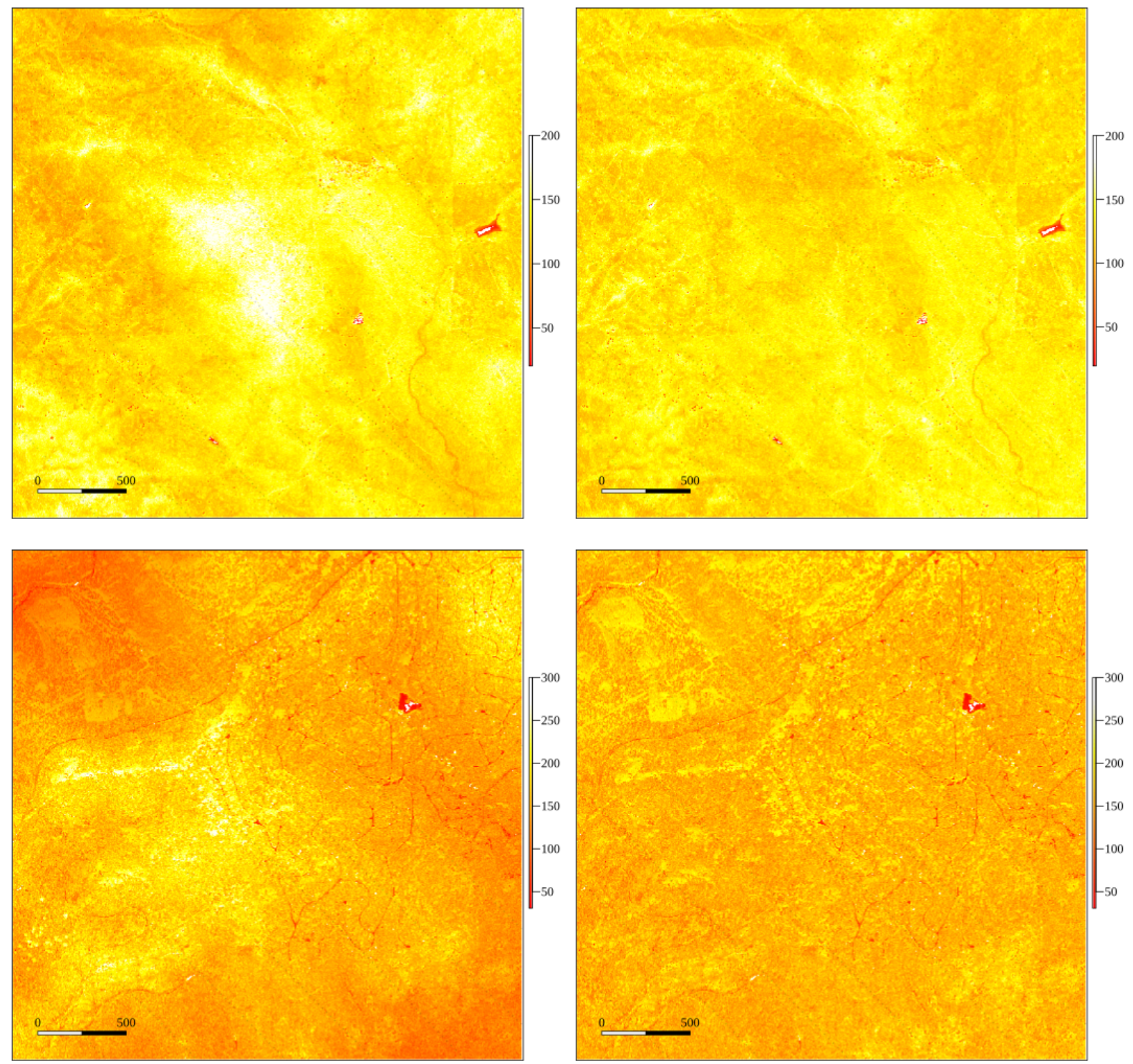

Figure 6: Images of the maximum intensity within $5 \times 5 \mathrm{~m}$ cells for raw intensities (left) and range-normalized intensities (right) for regions of interest \#5.1 and \#5.2 (table 1). Narrow and almost transparent lines correspond to the sensor trajectory reconstructed. On the left hand side, the effects of topography are clearly visible and one can see some obvious incoherence. On the right hand side these artifacts are no longer visible. Intensity correction also reveal land structure that were previously hidden in the noise generated by flightlines and topography.

the arbitrary value $f=2$ used in several studies (e.g. Yoga et al., 2017; Höfle and Pfeifer, 2007; García et al., 2010) was applied instead (results not shown). Compared to arbitrary values, using the optimal $f$ value had a measurable effect on the minimization of the differences in overlaps, but this effect remained small.

Gatziolis (2013) proposed that the range-correction should take each pulse into account. According to their results, the optimal value of $f$ depends not only on the position of the point in the return sequence but also on the pulse type, which implies that the intensity of pulses with different numbers of returns should be normalized using different $f$ values. They also suggested that the $f$ value must also be adapted as a function of the beam penetration within the canopy. They justify these variations of $f$ by the fact that multiple returns, or returns that penetrate deeper into the canopy, modify the range, which in turn causes artificial variation of computed $f$ values that may not be related to target properties.

Our results suggest that optimal values of $f$ also depended on the location chosen within a given dataset and on the specific derived metric that is tested (mean intensity, maximum intensity). The effect of location makes sense since we can expect local variations of land structures, 
and thus variation of target properties on average. The effect of the metrics can be interprets in relation to the results of Gatziolis (2013). Indeed, the maximum intensity is the result of a single point, likely from a high and large target with high reflectance, while the mean intensity is an aggregate of all the targets and all returns, which in a forested area implies deeper penetration into the canopy as well as an increase in structural complexity. Since each pulse should be corrected differently, we were expecting such variations. It remains, however, that the $f$ values reported by Gatziolis (2013) ranged between 2.0 and 3.0, and like in our study, most values remained between 2.1 and 2.6. While the work of Gatziolis (2013) is of great scientific value, we argue that for practical purposes, such variations can be accepted as part of the residual error of the model. Indeed, it would not only be impractical to apply range corrections calibrated for each subarea, forest type, or individual pulse type, but would also likely lead to over-interpretation. An important factor to keep in mind is that the sampled areas are made of different structures, and the reflectivity of each target is unknown, as well as their size, which prevents any possibility to retrieve the true physical meaning of the intensity values. Consequently, for practical applications of the range correction, there is no need to carefully select a precise value of $f$ different for each point. We therefore recommend using an $f$ value of 2.2 to 2.4 instead of the usual value of 2 over forested areas. The work of Gatziolis (2013) and figures 4, 5, and 6 as well as figures S1-S6 in supplementary materials suggest that values in this range should produce acceptable results on average, i.e. to efficiently remove the main effects of topography and sensor altitude variation; however, all the uncertainty related to the illuminated area of the targets and their reflectance will remain.

\subsection{Applications and limits of the tracking method}

Our sensor tracking method being based on tracing multiple returns, its applicability depends on the availability of vertically extended targets such as trees in forests. For bare ground areas such as deserts, low shrublands, high mountains, ice sheets or crop fields the method will likely fail. However, over forested areas, which represent a large part of the Earth's surface, the method is applicable as demonstrated in this study. In cities, building edges as well as urban trees and parks should provide more than enough multiple returns to perform the computation. Even over crop fields, it is unlikely that no trees or buildings can be found in a given scene. Retrieving the position of the aircraft every 0.5 seconds is not a requirement, because the positions are interpolated and aircraft routes are generally very stable. Thus, a decrease in the time resolution of the tracking, or even having missing points over several seconds should generally not represent a big issue. Our method should therefore be applicable over most landscapes, except in areas consisting of almost pure bare ground.
Aside from a range-based intensity correction, another potential application of our proposed tracking method would be to compute the real scan angle of each point relatively to nadir. The scan angle given in the . las file format (formats 0 to 5 ) is the scan angle rank i.e. an angle rounded as an integer that is relative to the sensor's self $0^{\circ}$ reference, which does not usually compensate for aircraft rolling. A scan angle computation from sensor positioning could therefore provide a roll-corrected angle of incidence with a resolution close to the arcminute for every individual point.

\subsection{Reproducibility}

One of our goals was to provide the research community an immediate way to reproduce this research. For this reason, we have provided algorithms in an easily usable form within the lidR package for R (Roussel and Auty, 2019), which is available on CRAN. The functions for sensor tracking and range correction are available from version 2.2.0 onward.

Because the lidR package was designed for real processing on broad coverage areas, computation time is an important issue to consider. Our least square intersection method is fast and can be computed under a second per square kilometre on a regular laptop. The key to the speed of the implemented calculation is that it only uses a small fraction of the points. By keeping one pulse every 0.0001 second and filtering out single returns, our computation was performed on less than one multiple return pulse per square meter. Using more points only increases linearly the computation time, for no significant gain in accuracy. Our method implementation is therefore suitable for large-scale processing. Similarly, the range correction was optimized to ensure a rapid computation time that is linearly dependent on the point density.

\section{Conclusion}

The lack of normalization is an issue that has hindered the use of intensity values in ALS data. Previous solutions have either relied on calibration data or on knowledge of the sensor trajectory, both of which are often non available. To resolve the issue, we have used an algorithm that can retrieve the sensor positioning at any time during the acquisition with an accuracy of a few meters. Using this tool, we were able to compute accurately a model-driven correction of range, which independently reproduced and validated the previous work of Gatziolis (2013) as well as confirming the reproducibility of the results. In view of our results and previous results cited in this study, we recommend using a power $f$ between 2.1 and 2.3 in the equation of range correction instead of the usual value of 2 in a forested context, but this adjustment is not critical. One key feature of our method is that it does now require any additional data. With the source code being public and ready-to-use in the $\mathrm{R}$ environment, we believe that our study provides a basis for further intensity-based research using ALS data. 


\section{Acknowledgement}

Data for northern Vancouver Island was generously supplied by Western Forest Products Inc. and BC Timber Sales. Data for California was generously supplied publicly by the U.S. Geological Survey (USGS). Data of sensor position was generously supplied by the Ministère des Forêts, de la Faune et des Parcs of Québec.

\section{References}

Baltsavias, E. (1999). Airborne laser scanning: basic relations and formulas. ISPRS Journal of Photogrammetry and Remote Sensing, 54(2-3):199-214.

Bright, B. C., Hudak, A. T., McGaughey, R., Andersen, H. E., and Negrón, J. (2013). Predicting live and dead tree basal area of bark beetle affected forests from discrete-return lidar. Canadian Journal of Remote Sensing, 39(SUPPL.1):99-111.

Dickersin, K. (1990). The existence of publication bias and risk factors for its occurrence. Jama, 263(10):1385-1389.

Donoghue, D., Watt, P., Cox, N., and Wilson, J. (2007). Remote sensing of species mixtures in conifer plantations using LiDAR height and intensity data. Remote Sensing of Environment, 110(4):509-522.

García, M., Riaño, D., Chuvieco, E., and Danson, F. M. (2010). Estimating biomass carbon stocks for a Mediterranean forest in central Spain using LiDAR height and intensity data. Remote Sensing of Environment, 114(4):816-830.

Gatziolis, D. (2013). Dynamic Range-based Intensity Normalization for Airborne, Discrete Return Lidar Data of Forest Canopies. Photogrammetric Engineering \& Remote Sensing, 77(3):251-259.

Höfle, B. and Pfeifer, N. (2007). Correction of laser scanning intensity data: Data and model-driven approaches. ISPRS Journal of Photogrammetry and Remote Sensing, 62(6):415-433.

Hopkinson, C. (2007). The influence of flying altitude, beam divergence, and pulse repetition frequency on laser pulse return intensity and canopy frequency distribution. Canadian Journal of Remote Sensing, 33(1-4):312-324.

Kaasalainen, S., Hyyppä, J., Litkey, P., Hyyppä, H., Ahokas, E., Kukko, A., and Kaartinen, H. (2007). Radiometric calibration of als intensity. Int. Arch. Photogramm. Remote Sens, 36:201-205.

Kim, S., McGaughey, R. J., Andersen, H.-E., and Schreuder, G. (2009). Tree species differentiation using intensity data derived from leaf-on and leaf-off airborne laser scanner data. Remote Sensing of Environment, 113(8):1575-1586.

Korpela, I., Tokola, T., Ørka, H. O., and Koskinen, M. (2009). Small-footprint discrete-return LIDAR in tree species recognition. In Proceedings of the ISPRS, number 2004, pages 2-5.

Korpela, I. S. (2008). Mapping of understory lichens with airborne discrete-return lidar data. Remote Sensing of Environment, 112(10):3891-3897.

Liu, X. (2008). Airborne lidar for dem generation: some critical issues. Progress in Physical Geography, 32(1):31-49.

Moffiet, T., Mengersen, K., Witte, C., King, R., and Denham, R. (2005). Airborne laser scanning: Exploratory data analysis indicates potential variables for classification of individual trees or forest stands according to species. ISPRS Journal of Photogrammetry and Remote Sensing, 59(5):289-309.

Popescu, S. C. and Zhao, K. (2008). A voxel-based lidar method for estimating crown base height for deciduous and pine trees. Remote Sensing of Environment, 112(3):767-781.

Roussel, J.-R. and Auty, D. (2019). lidR: Airborne LiDAR Data Manipulation and Visualization for Forestry Applications. R package version 2.1.0.

Sumnall, M. J., Hill, R. A., and Hinsley, S. A. (2016). Comparison of small-footprint discrete return and full waveform airborne lidar data for estimating multiple forest variables. Remote Sensing of Environment, 173:214-223.

U.S. Geological Survey (2011). 20140929, USGS Lidar Point Cloud (LPC) CA_CalaverasTuolumne_2011_001504 2014-08-27 LAS: U.S. Geological Survey.
Vain, A., Kaasalainen, S., Pyysalo, U., Krooks, A., and Litkey, P. (2009). Use of Naturally Available Reference Targets to Calibrate Airborne Laser Scanning Intensity Data. Sensors, 9(4):2780-2796.

Vain, A., Kaasalainen, S., and Wang, C. (2011). Correcting airborne laser scanning intensity data. Laser Scanning, Theory and Applications, pages 49-60.

Wagner, W. (2010). Radiometric calibration of small-footprint full-waveform airborne laser scanner measurements: Basic physical concepts. ISPRS Journal of Photogrammetry and Remote Sensing, 65(6):505-513.

Wing, B. M., Ritchie, M. W., Boston, K., Cohen, W. B., and Olsen, M. J. (2015). Individual snag detection using neighborhood attribute filtered airborne lidar data. Remote Sensing of Environment, 163:165-179.

Yao, W., Krzystek, P., and Heurich, M. (2012). Identifying Standing Dead Trees in Forest Areas Based on 3D Single Tree Detection from Full Waveform LIDAR Data. ISPRS Annals of Photogrammetry, Remote Sensing and Spatial Information Sciences, I-7(September):359-364.

Yoga, S., Bégin, J., St-Onge, B., and Gatziolis, D. (2017). Lidar and multispectral imagery classifications of balsam fir tree status for accurate predictions of merchantable volume. Forests, 8(7).

Yunfei, B., Guopingb, L., and Chunxiang, C. (2008). Classification of LIDAR point cloud and generation of DTM from LIDAR height and intensity data in forested area. ISPRS Congress, (August):313-318.

Zhang, Z. and Liu, X. (2013). Support vector machines for tree species identification using LiDAR-derived structure and intensity variables. Geocarto International, 28(4):364-378. 